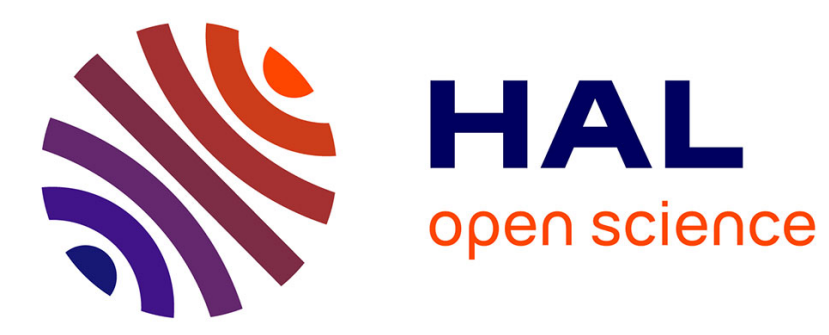

\title{
Voix croisées : la compilation du Shinkokin waka shû à travers les témoignages de deux protagonistes
}

\author{
Michel Vieillard-Baron
}

\section{To cite this version:}

Michel Vieillard-Baron. Voix croisées : la compilation du Shinkokin waka shû à travers les témoignages de deux protagonistes. Extrême-Orient Extrême-Occident, 2003, L'anthologie poétique en Chine et au Japon, 25 (25), pp.55-80. 10.3406/oroc.2003.1165 . hal-01314373

\section{HAL Id: hal-01314373 \\ https://hal-inalco.archives-ouvertes.fr/hal-01314373}

Submitted on 11 May 2016

HAL is a multi-disciplinary open access archive for the deposit and dissemination of scientific research documents, whether they are published or not. The documents may come from teaching and research institutions in France or abroad, or from public or private research centers.
L'archive ouverte pluridisciplinaire HAL, est destinée au dépôt et à la diffusion de documents scientifiques de niveau recherche, publiés ou non, émanant des établissements d'enseignement et de recherche français ou étrangers, des laboratoires publics ou privés.

\section{(ㅇ)(1) $\$$}

Distributed under a Creative Commons Attribution - NonCommercial - NoDerivatives| 4.0 


\section{Voix croisées : la compilation du Shinkokin waka shû à travers} les témoignages de deux protagonistes

Michel Vieillard-Baron

\section{Citer ce document / Cite this document :}

Vieillard-Baron Michel. Voix croisées : la compilation du Shinkokin waka shû à travers les témoignages de deux protagonistes. In: Extrême-Orient, Extrême-Occident, 2003, n²5. L'anthologie poétique en Chine et au Japon. pp. 55-80. doi : 10.3406/oroc.2003.1165

http://www.persee.fr/doc/oroc_0754-5010_2003_num_25_25_1165

Document généré le 16/10/2015 


\title{
Résumé
}

Voix croisées : la compilation du Shinkokin waka shû à travers les témoignages de deux protagonistes

Depuis qu'en 905 l'empereur Daigo commandita le Kokin waka shû, la production d'une anthologie de poèmes japonais constituait l'un des symboles les plus éclatants du règne d'un monarque. Vingt et une anthologies impériales furent produites jusqu'en 1439. Dans le présent article, nous décrivons la compilation de la huitième intitulée Shinkokin waka shû. Cette dernière fut ordonnée en 1201 et achevée en 1216. Pour connaître la manière dont fut compilée l'anthologie, nous disposons de deux documents importants et complémentaires : le lenaga nikki, mémoires de Minamoto no lenaga, secrétaire du Bureau de la poésie, chargé des affaires liées à la compilation, et surtout le Meigetsuki, notes journalières de Fujiwara no Teika, célèbre poète qui fut également I'un des principaux compilateurs. En croisant ces deux témoignages, on prend pleinement conscience du processus long et harassant qui aboutit à l'un des chefs-d' oeuvres les plus parfaits de la littérature japonaise.

\begin{abstract}
Narrating Voices : the Compilation of the Shinkokin waka shû as described in the Accounts of Two Witnesses

Ever since Emperor Daigo ordered the compilation of the Kokin waka shû in 905, an imperial collection of Japanese poetry was considered an important symbol of a monarch's reign. Twentyone imperial anthologies were compiled, the last completed in 1439. In this paper, I describe the compilation of the Shinkokin waka shû, the eighth imperial collection, which was commissioned in 1201 and completed in 1216. In order to reconstruct the process, I have used two major complementary sources : the lenaga nikki, the memoir of Minamoto lenaga, secretary of the office that provided research and editing support for the anthology, and the Meigetsuki, the diary of the famous poet Fujiwara Teika, one of the principal compilers. By examining these two texts in tandem, we can more fully grasp the long and arduous process that produced one of the most perfect masterpieces of Japanese literature.
\end{abstract}




\title{
Voix croisées : la compilation du Shinkokin waka shû à travers les témoignages de deux protagonistes
}

\author{
Michel Vieillard-Baron
}

Le 11 du premier mois de l'année 1198, l'empereur Gotoba, jeune homme de dix-huit ans, abdiqua au profit de son fils, l'empereur Tsuchimikado, âgé de trois ans; on l'appela dès lors «l'empereur retiré Gotoba», alias Gotobain. Cette abdication permettait à Gotoba de conserver les rênes du pouvoir sans avoir à assumer, entre autres, les nombreuses et harassantes célébrations qui rythmaient la vie de la Cour. Elle lui permettait en outre - et surtout - de consacrer davantage de temps à ses deux passions: le sport ${ }^{1}$ et la poésie. Profitant de son nouveau statut, Gotoba organisa bientôt des manifestations qui virent s'affronter les meilleurs poètes de son temps. Avec un art consommé, il sut combiner et faire rivaliser des poètes aux sensibilités très différentes, suscitant une émulation sans précédent. Gotoba, conscient de l'exceptionnel potentiel du cercle poétique qui s'était constitué autour de lui, eut vraisemblablement bientôt l'idée, sans tout d'abord révéler son dessein, d'ordonner ce qui, dans la tradition japonaise, symbolise le plus parfaitement le règne éclairé d'un empereur: la compilation d'une anthologie impériale ${ }^{2}$. Aussi, en 1201, ressuscita-t-il un office depuis longtemps disparu, le Bureau de la poésie, wakadokoro. Cet office, calqué sur celui établi à l'époque de l'empereur Murakami en 951 pour la compilation de la deuxième anthologie impériale, devait traiter toutes les questions concernant les activités poétiques organisées par Gotoba, et en particulier la compilation de notre anthologie. Précisons, avant d'aller plus avant dans notre étude, que les anthologies qui nous occupent contiennent exclusivement - ou essentiellement - des waka, «poèmes japonais» de 31 syllabes réparties en cinq vers ou $k u$ de $5,7,5,7$, 7 syllabes respectivement ${ }^{3}$; c'est de ce type de poème qu'il s'agira ici.

Pour connaître la façon dont fut compilée cette anthologie intitulée Shinkokin waka shû / Nouveau recueil de poèmes anciens et modernes (titre parfois abrégé en Shinkokin, ou Shinkokin shû) nous disposons de deux témoignages exceptionnels et complémentaires: le Ienaga nikki / Mémoires 
d'Ienaga ${ }^{4}$ et le Meigetsuki / Notes de la lune claire ${ }^{5}$. Le lenaga nikki est l'œuvre de Minamoto no lenaga (?-1234), le secrétaire du Bureau de la poésie. Ce texte, écrit en japonais, fut sans doute rédigé entre 1216 et $1221^{6}$, c'est-à-dire après les événements qu'il relate ${ }^{7}$. Ienaga écrit dans un style qui se veut proche de celui qu'employaient les dames de la Cour pour écrire leurs mémoires, un style à la fois élégant, fluide mais souvent ambigu. Le but d'Ienaga était de dresser un monument à la gloire de Gotoba; la valeur de ce document se trouve donc en partie diminuée par le manque d'objectivité de son auteur. Il n'en demeure pas moins que la position centrale d'lenaga en tant que secrétaire du Bureau de la poésie rend son texte précieux pour connaître ce que l'on pourrait appeler la «voix officielle» de la Cour. Pour contrebalancer ce texte souvent vague et laconique, nous convoquerons également le Meigetsuki, les notes journalières de Fujiwara no Teika (11621241). Teika, l'un des plus grands poètes de ce temps ${ }^{8}$, faisait partie du Bureau de la poésie et du comité de compilation de l'anthologie impériale, au sein duquel il devait jouer un rôle essentiel. Il rédigea ses notes journalières en chinois, suivant en cela l'usage de ce temps'. Contrairement au lenaga nikki qui se présente comme une œuvre littéraire, publique, le Meigetsuki était destiné à l'usage exclusif des proches de Teika, de sa famille; il s'agit donc d'un document privé. Dans ce texte, écrit au jour le jour, Teika n'hésite pas à décrire avec une grande franchise les événements, petits et grands, de sa vie. On sera frappé par le contraste entre les descriptions approximatives et d'une imprécision souvent irritante d'Ienaga et celles d'une minutie presque maniaque de Teika ${ }^{10}$. Ces hommes aux personnalités très différentes ont tous deux pris part à la compilation du Shinkokin waka shû qu'ils ont racontée, chacun à sa manière. Il s'agira ici de les écouter, de croiser leurs témoignages, pour comprendre ce que fut l'aventure de la compilation de la huitième anthologie impériale.

Le premier pas dans le processus de compilation de l'anthologie fut l'instauration par Gotoba d'un Bureau de la poésie. Cet office fut installé dans sa résidence privée, le palais Nijô, qui se trouvait au croisement des avenues Nijô et Higashi no Tô.in, dans la capitale Heian-kyô (l'actuelle Kyôto). Nous savons grâce aux notes journalières de Teika que le Bureau de la poésie se trouvait au Nord du pavillon Hirogosho ". Ce pavillon avait une surface d'environ 324 mètres carrés (neuf travées du nord au sud et quatre travées d'est en ouest); le Bureau de la poésie, situé à l'extrême nord du pavillon, occupait quelque 36 mètres carrés (six travées de long sur deux travées de large). 
Voix croisées: la compilation du Shinkokin waka shû

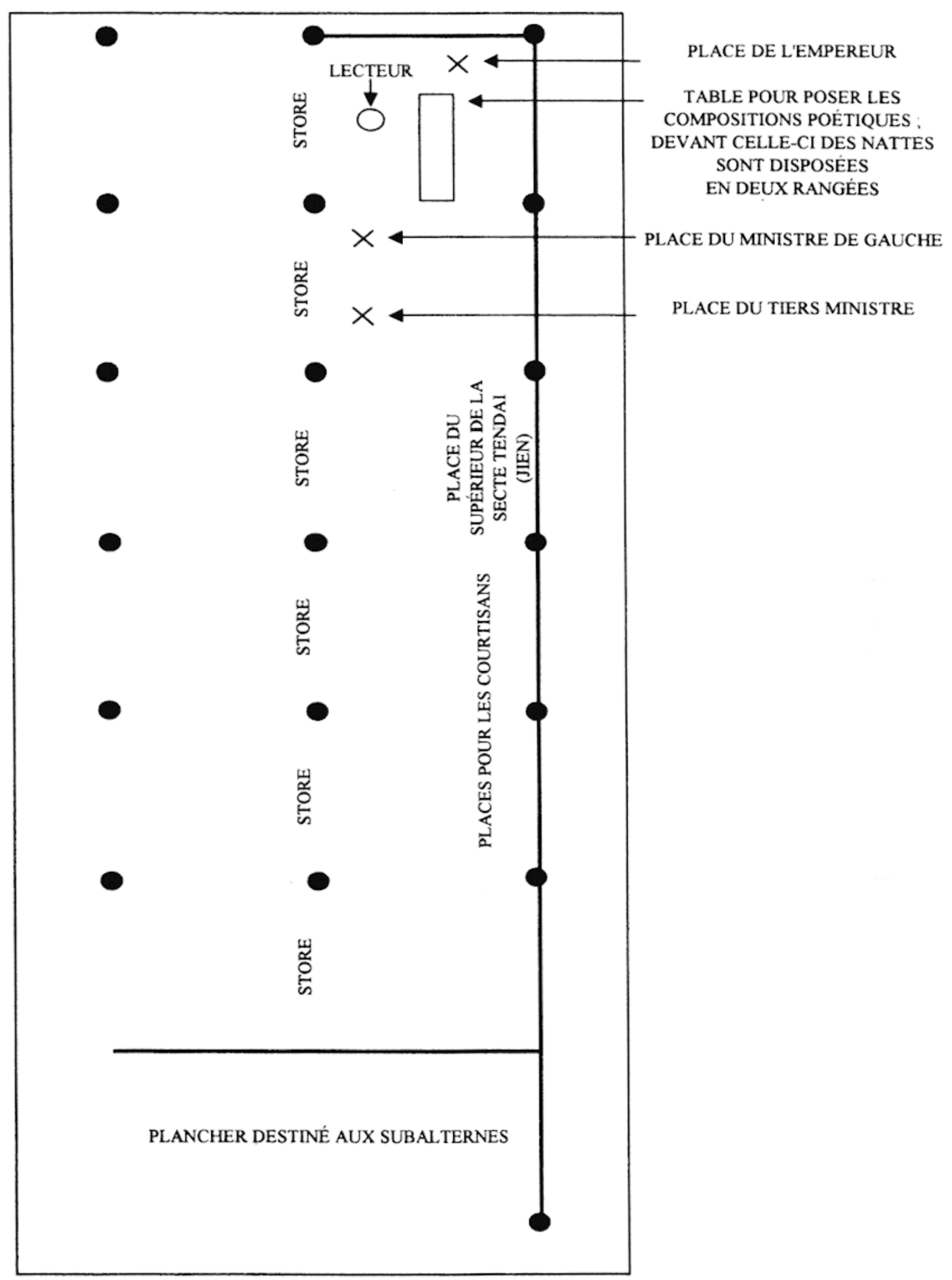

Plan du Bureau de la poésie d'après le schéma tracé par Teika dans ses notes journalières (Kennin 1 [1202], VII, 27). 
Laissons à présent la parole à lenaga :

En cette année que le nom d'ère devint Kennin (c.-à-d. en 1201), fut instauré le Bureau de la poésie. Afin de le loger, le pavillon Hirogosho du Palais Nijô fut transformé. Sur une largeur de deux intervalles (c.-à-d. six mètres), le plancher fut rabaissé pour constituer l'espace où siégeraient les courtisans ${ }^{12}$, pour les autres, un plancher placé à niveau [était prévu]. Les personnes nommées au Bureau de la poésie étaient: le régent ministre de gauche ${ }^{13}$ [Yoshitsune], le tiers ministre Michi [chika] ${ }^{14}$, Ariie ason ${ }^{15}$, Michitomo ason, Ietaka, Teika ason, Tomochika, Masatsune, le moine Jakuren, le moine Shakua ${ }^{16}$. Le premier jour que je me rendis au Bureau de la poésie après avoir été nommé secrétaire ${ }^{17}$, je présentai ce poème:

Moshihogusa

Kakutomo tsukiji

Kimi ga yo no

Kazu ni yomi woku

Waka no ura nami
Les algues à sel

Malgré la récolte, toujours abondent

De même, durant Votre vie

D'innombrables poèmes nous composerons:

Vagues dans la Baie de la Waka ${ }^{18}$

Vêtu convenablement de mon costume de Cour, j'allai au Bureau de la poésie où je notai ce poème et le présentai à l'Empereur retiré en me conformant aux règles de protocole habituelles. Au départ, seules les personnes mentionnées ci-dessus [faisaient partie du Bureau de la poésie], mais par la suite, [le courtisan] Takanobu ason et les subalternes Kamo no Chômei et Fujiwara no Hideyoshi furent également nommés $[\ldots]^{19}$.

lenaga oublie de mentionner dans sa liste le moine Jien (1155-1225), supérieur général de l'école bouddhique Tendai qui fut nommé parmi les premiers membres du Bureau de la poésie ${ }^{20}$. Quatorze personnes, choisies pour leur talent poétique, constituaient donc le personnel de ce Bureau. Il s'agissait là, comme le prouve la citation suivante, d'un travail à plein temps fort contraignant :

Les membres se retrouvaient au Bureau de la poésie où chacun signait le registre de présence, et, à la fin de chaque mois, présentait à l'Empereur retiré son état de service mensuel; chacun rivalisait alors pour avoir le plus grand nombre de jours de présence ${ }^{21}$. Qu'il neige, que la lune [soit particulièrement belle] ou les cerisiers en fleur, [peu importait], les membres se rendaient [au Bureau de la poésie] même quand il n'y avait pas de manifestation [particulière]. Lorsque l'Empereur retiré n'était pas présent, souvent, tout naturellement ils composaient à titre personnel, sans raison particulière. C'est ainsi que ce Bureau a permis d'éviter le déclin de la voie de la poésie. C'était [également] le lieu d'expériences poétiques. [Ainsi, un jour,] plus de vingt personnes étaient rassemblées ${ }^{22}$ : le régent ministre de gauche (Yoshitsune) et le tiers ministre (Michichika) siégaient sur la galerie à l'est; les autres participants étaient alignés sur le vaste plancher, face à l'est, la place d'honneur. De derrière un store en bambou, Gotoba donna les sujets de dix poèmes. Comme tous les participants ont composé sur ces sujets, je ne consigne 
pas les poèmes ici. Certains membres ont sans doute été particulièrement brillants et furent sollicités pour produire des poèmes [en d'autres occasions]. Les membres du Bureau de la poésie étaient pratiquement toujours en service, et comme les manifestations poétiques se suivaient à un rythme soutenu, je n'ai pas pu toutes les consigner. Je pense toutefois que j'aurais dû noter - même dans leurs grands traits - les poèmes qui furent par exemple particulièrement remarqués, mais j'avais alors trop de tâches publiques et privées à mener à bien et je ne les ai pas transcrits. Quand plus tard les gens me poseront des questions, je me dirai: «Quel dommage! », mais comme les participants ont sans doute à présent dispersé leurs notes et qu'il serait difficile de les retrouver, il ne me reste plus qu'à me résigner. Je regrette d'oublier même certaines choses dont pourtant je me souvenais ${ }^{23}$.

Les membres du Bureau de la poésie étaient donc des employés dont la tâche principale consistait pour lors à participer aux nombreuses manifestations poétiques organisées par Gotoba ${ }^{24}$. L'empereur retiré savait que la compilation d'une anthologie nécessitait la production d'un important corpus de poèmes dans lequel les personnes chargées de la sélection pourraient puiser, il multiplia donc les manifestations :

[Les manifestations poétiques] se succédaient comme à l'accoutumée, bons et mauvais poèmes s'empilaient en grand nombre. En outre, comme parmi les compositions de jadis il y en avait certainement [d'intéressantes] qui avaient échappé à la vigilance des compilateurs [des anthologies impériales précédentes], l'Empereur retiré ordonna à six personnes de les rechercher et de les rassembler en y mettant toute l'ardeur dont ils étaient capables, afin de les lui présenter. Ces personnes étaient: Michitomo ason, Ariie ason, Teika ason, Ietaka, Masatsune, et le moine Jakuren. Tous acceptèrent et partirent à la recherche [de bons poèmes], rivalisant afin de ne laisser aucun interstice inexplore «du sommet de la plus haute montagne aux tréfonds de la mer». Il ne pouvait cependant être question de retenir tous les poèmes que ces hommes avaient choisis et présentés: ils devaient tout d'abord être examinés par l'Empereur retiré. Aussi, tous ceux qui en ce bas monde se considéraient comme des poètes, s'en allèrent le cœur battant prier dieux et bouddhas pour que leurs poèmes fussent retenus ${ }^{25}$.

C'est en Kennin 1 (1201), le 3 du onzième mois, que Gotoba donna l'ordre à six membres du Bureau de la poésie, dont Teika, de lui présenter des poèmes anciens et modernes qui pourraient faire l'objet d'une sélection. Cette date scelle la commande officielle de l'anthologie impériale dont les six hommes devenaient les compilateurs, endossant ainsi la fonction la plus prestigieuse qui puisse être confiée à un poète. Procéder à une première sélection de poèmes - qui allait occuper l'essentiel de l'année 1202 (Kennin 2) représentait pour les six compilateurs un travail de Titan; le corpus était colossal (plusieurs centaines de milliers de poèmes sans doute), et les compilateurs devaient veiller à ne pas sélectionner de poèmes qui avaient déjà été 
retenus dans les sept anthologies impériales précédentes, leur réutilisation étant en principe proscrite. Dans ses notes journalières (Kennin 2,1202, III, 18) Teika écrit : «En ce moment, je passe mes journées à rassembler d'anciens poèmes; je ne fais rien d'autre [...]», ce qui devait être le lot de tous les compilateurs.

Le septième mois de cette même année 1202 meurt Jakuren, l'un des compilateurs; il ne sera jamais remplacé. Jakuren était le demi-frère de Teika et sa mort l'affecta profondément comme l'atteste ce passage de ses notes journalières (Kennin 2, VII, 20):

Ayant entendu cela, je quitte aussitôt le palais. [...] Que la vie soit une chose si fragile, impermanente, ne me surprend pas, mais en apprenant cette nouvelle je n'ai pu m'empêcher d'éprouver de la tristessc ct d'éclater en sangluts. Depuis notre lointaine enfance nous avons été proches, et cela remonte à plusieurs dizaines d'années. En outre, dans la voie de la poésie, qui peut rivaliser avec lui? Sa mort est une grande perte pour la poésie $[\ldots]^{26}$.

Malgré la mort de Jakuren, le travail de sélection devait continuer. Les compilateurs poursuivaient le fastidieux travail consistant à passer en revue tous les recueils en leur possession ainsi que les procès-verbaux de manifestations poétiques afin d'en retenir les poèmes les plus intéressants. Ils recevaient en outre des œuvres et des suppliques de personnes souhaitant voir un de leurs poèmes retenu dans la prestigieuse anthologie. Teika décrit dans son Meigetsuki l'effet désastreux de ce travail sur sa santé:

Kennin 2 (1202), VIII, 13: Depuis hier, mon oil droit est très enflé: ai-je épuisé mes yeux à sélectionner des poèmes? Pendant deux jours je me suis rendu à mon travail en me ménageant, mais depuis la nuit dernière, l'enflure est de la grosseur d'un crapaud. À cause de cela je ne me rends pas à mon travail ${ }^{27}$.

Le 20 du dixième mois de cette même année Kennin 2 (1202), meurt Minamoto no Michichika qui élait membre du Bureau de la poésie mais ne faisait pas partie des compilateurs. Ces derniers, faisant face à l'adversité, poursuivaient inlassablement leur travail, demandant toutefois à l'occasion - comme en témoigne Teika -, le soutien des dieux :

Kennin 3 (1203), III, 29: De bon matin je me rends au sanctuaire de Kitano afin de prier pour la sélection des poèmes; je fais offrande de papiers votifs et rentre à la maison.

Le 11 du quatrième mois de Kennin 3 (1203), Ienaga fait dire à Teika qu'il doit présenter sa sélection de poèmes à Gotoba avant le 20 du mois. Teika, qui n'a, nous dit-il, «rien fait d'autre ces vingt derniers jours et nuits que lire d'anciens poèmes ${ }^{28}$ », termine «tout juste» dans la nuit du 19 au 20 de copier 
sa sélection, y jette un dernier coup d'œil le matin et la porte au palais vers midi ${ }^{29}$. Ayant reçu les sélections de chacun des compilateurs, Gotoba va consacrer plus d'une année - jusqu'au sixième mois de l'année Genkyû 1 (1204) - à les réviser. Entre-temps, le 23 du onzième mois, l'empereur donna une fête en l'honneur du quatre-vingt-dixième anniversaire de l'un des membres du Bureau de la poésie, Shunzei ${ }^{30}$ - désigné le plus souvent dans nos textes par son nom religieux Shakua. Shunzei (1114-1204) - le père de Teika - avait compilé seul la septième anthologie impériale, arbitré de nombreux concours de poèmes et produit une œuvre d'une qualité remarquable : il était de ce fait considéré comme la plus grande autorité de ce temps en matière de poésie. Une semaine après cette manifestation - tout a fait exceptionnelle pour un courtisan d'un rang relativement modeste - , le grand poète s'éteignit ${ }^{31}$.

Le 20 du septième mois de la première année de Genkyû (1204), Gotoba ordonna aux compilateurs de procéder à la classification des poèmes par thème (burui). Teika écrit dans ses notes journalières :

Genkyâ 1 (1204), VII, 22 : À la tombée de la nuit, forte pluie et un peu de neige. Aujourd'hui on nous a donné l'ordre de nous rendre au Bureau de la poésie afin de commencer le classement des poèmes sélectionnés, travail initié il y a deux jours. J'y suis donc allé. Nous avons encore reçu des récriminations des moines du Mont Hiei [concernant l'inclusion de leurs poèmes] [...].

Le travail de classement par thèmes était une tâche délicate. Dans un premier temps, les compilateurs devaient répartir selon les différentes sections de l'anthologie les poèmes qu'ils avaient rassemblés. Ces différentes sections étaient, à quelques différences près ${ }^{32}$, celles de la première anthologie impériale: Printemps, Été, Automne, Hiver, Félicitations, Affliction, Voyages, Amour, Sujets divers, Shintô, Bouddhisme. Dans un second temps, ils devaient procéder au classement des poèmes au sein d'une même section. Il s'agissait là d'un travail extrêmement méticuleux car on devait percevoir à la lecture la progression du thème. Ainsi, au début de l'anthologie, le premier poème de la section Printemps, annonce-t-il l'arrivée de la saison, les suivants son passage, son zénith puis son déclin; le dernier poème de cette section chante, logiquement, le dernier jour de la saison. Ce travail qui consiste à intégrer des poèmes d'origine diverses en un tout parfaitement cohérent - et dont notre anthologie est le résultat le plus abouti - n'est pas sans rappeler la tâche des mosaïstes fixant une à une les petites pièces rapportées de manière à produire un motif ${ }^{33}$. Les poètes procédaient par étapes, affinant progressivement leur travail qui était consigné par un secrétaire de séance. Teika relate dans ses notes journalières le travail de classement: 
Genkyû 1 (1204), VII, 23: Étant chargé de continuer à classer les poèmes [au Bureau de la poésie], je ne me suis pas rendu à Minase [le lieu de villégiature de Gotoba]. J'ai toutefois rendu visite à Ienaga et Kiyonori qui m'ont dit que tout le monde y était allé. L'Empereur retiré est parti quant à lui vers deux heures de l'après-midi.

Genkyû 1 (1204), VII, 27 : Ienaga est rentré hier soir [de Minase]. À sa demande, je me rends au Bureau de la poésie. Là, je retrouve Michitomo ${ }^{34}$, Ariie, Masatsune et Ienaga. Nous ouvrons la boîte contenant les poèmes et nous mettons à les classer. Masatsune est chargé de consigner [notre travail]. Nous parvenons à achever la mise en forme des deux volumes de poèmes de printemps ${ }^{35}$. Le soir nous nous retirons.

Au cours de ces séances de travail qui devaient être éprouvantes, les compilateurs s'accordaient parfois des récréations :

Genkyû 1 (1204), VII, 28: De bon matin je me rends chez Son Excellence Yoshitsune $[\ldots]$ puis au Bureau de la poésie où nous nous retrouvons comme hier. letaka nous rejoint (Masatsune arrive particulièrement en retard). Michitomo apporte deux boîtes (sur lesquelles est inscrit Anthologie impériale $I$ et $I$ ). Une de ces boîtes, compartimentée, contient du melon; la seconde, en porcelaine, renferme entre autres du vin. Il y a également de la glace; Michitomo la débite avec son sabre, [le voir faire] est extrêmement captivant. Bien que nous nous soyons retirés au frais, nous ne sommes pas complètement isolés [et quelqu'un aurait pu le faire pour nous] mais Michitomo s'en charge et montre ainsi son adresse. Il enveloppe ensuite la glace dans un linge blanc et la concasse à l'aide de sa main gauche. Il fait cela sans la moindre difficulté, comme s'il avait été Gaoyao, [le sévère ministre de la justice de Shun, souverain mythique Chinois]. Tous en mangent et se régalent. Aujourd'hui nous avons terminé la section [des poèmes] d'été. Nous avons en outre consigné les poèmes de Tanabata ${ }^{36}$. Après avoir rangé, nous nous retirons $[\ldots]$.

À partir du huitième mois les compilateurs sont épuisés :

Genkyû 1 (1204), VIII, 7: Vers dix heures du matin je vais [chez Yoshitsune] et ensemble, nous nous rendons chez l'Empereur retiré. Bien qu'on nous ait ordonné hier de poursuivre le travail de classification, personne n'est venu, se déclarant malade $[\ldots]$.

Teika montre même des signes de dépression nerveuse, liée sans doute à l'épuisement physique et intellectuel ainsi qu'à ses problèmes de santé récurrents:

Genkyû 1 (1204), IX, 24 : [...] Depuis que je suis rentré à la maison, ma maladie me fait particulièrement souffrir [...]. Bien qu'il y ait ces derniers jours des séances quotidiennes de classification de poèmes, je fais dire qu'il n'y a pas de 
remède à ma maladie [et ne m'y rends pas]. Je n'ai plus goût à rien; la vie publique me semble extrêmement stérile. La voie [de la poésie] n'a plus d'intérêt pour moi ; les semaines et les mois, je les passe à me forcer. Tout cela ne fera-t-il qu'augmenter ma honte?

Malgré l'accablement des compilateurs, Gotoba poursuit sans le moindre état d'âme l'objectif qu'il s'est fixé: mener à bien dans les meilleurs délais l'achèvement de son anthologie. Il réussit ainsi à obtenir un premier état du Shinkokin waka shû. Bien que l'anthologie soit encore dépourvue de préfaces (elle devait en avoir deux, l'une en chinois, l'autre en japonais) ${ }^{37}$, et que le classement des poèmes soit encore loin d'être achevé, Gotoba, impatient, décide d'organiser un banquet le 26 du troisième mois de Genkyû 1 (1204) pour fêter la fin de la compilation. Laissons à présent la parole à Ienaga:

Une fois le classement par thème des poèmes du Shinkokin achevé, il y eut au quatrième mois $^{38}$ [un banquet] ${ }^{39}$. L'Empereur retiré, inquiet [du temps que prendrait l'achèvement complet du travail], décida de donner ce banquet alors même que l'anthologie était encore dépourvue de préfaces ${ }^{40}$. Depuis le dixième mois ${ }^{41}$ de l'année dernière (1204), les compilateurs se réunissaient au Bureau de la poésie et, de huit heures du matin à la tombée de la nuit, écrivaient, supprimaient, rajoutaient des poèmes sans le moindre répit, au point d'en avoir les mains engourdies. [L'Empereur retiré] se montrait des plus tatillons dans le choix des poèmes. Une fois que chacun des cinq compilateurs lui avait montré sa sélection, l'Empereur retiré la lisait entièrement et marquait d'un signe les poèmes qu'il considérait intéressants. Le capitaine de la garde du corps section de gauche Kiyonori recopiait alors ces derniers au propre et les présentait à Gotoba qui les examinait de nouveau, les faisant recopier jusqu'à trois fois [dans certains cas]. Vraiment, que l'auteur fût puissant ou misérable, brillant ou sot, peu importait; seule comptait la qualité du poème. Ainsi, dans le cas d'une personne de peu d'importance - s'agissant même du moine d'un temple de montagne -, si celle-ci montrait du talent dans la voie de la poésie, naturellement son poème devait être retenu. Les cinq compilateurs rassemblèrent les poèmes que l'Empereur retiré avait marqués sur chacun des manuscrits et s'efforcèrent de les classer. [La quantité de papier était telle] que le Bureau de la poésie leur sembla l'endroit le plus exigu qui soit ${ }^{42}$.

Pressé de voir son œuvre aboutir, Gotoba ne laisse plus aucun répit à ses compilateurs et prend lui-même une part extrêmement active au travail de sélection. Aucun empereur avant lui ne s'était autant impliqué dans la compilation de ce type d'anthologie. L'investissement personnel de Gotoba est tel qu'Ienaga écrit:

L'Empereur retiré avait tant de fois lu et relu les deux mille poèmes que contient l'anthologie qu'il les connaissait par cœur et qu'il pouvait les réciter avec une 
exactitude exceptionnelle. Bien qu'il ne nous soit pas venu à l'esprit de mettre en doute la réalité de ses capacités, l'Empereur retiré nous engagea à le mettre à l'épreuve nous disant: «Prenez deux ou trois livres de poèmes que vous avez déjà classés et lisez-moi le premier vers de n'importe laquelle des compositions et je vous en réciterai la fin". Nous prîmes alors discrètement l'un des livres et lûmes le premier vers de certains poèmes que l'Empereur retiré complétait sans jamais se tromper. Il était sans doute logique qu'il en fût ainsi puisqu'il lui suffisait de lire un poème deux ou trois fois pour le mémoriser sans jamais l'oublier. À plus forte raison, [dans le cas de cette anthologie], il lui était arrivé à de nombreuses reprises d'avoir à se prononcer sur la qualité ou les défauts d'un poème dans la perspective de sa sélection, aussi est-il naturel qu'il les ait tous conservés en mémoire. Cette anthologie devant être [d'une qualité] sans précédent, les poètes qui en avaient conscience et dont les œuvres avaient été écartées, utilisaient toutes leurs relations ou bien encore écrivaient des lettres de requêtes, usant jusqu'à la dernière les plus belles formules, et joignaient de nouvelles compositions; les demandes affluaient de toutes parts, plus nombreuses que des gouttes de pluie. À cette époque, Gotoba négligeait les affaires relatives au gouvernement et ne se préoccupait que de la compilation de l'anthologie; aussi, les membres de la chancellerie privée et les employés attachés au service de l'Empereur retiré discutaient et se disaient qu'ils avaient bien du temps libre. Comme un membre de la chancellerie se plaignait qu'il n'y avait rien d'exceptionnel à faire puisqu'il ne se passait rien d'important, Gotoba lui répondit: «En ce moment, je ne puis entendre vos récriminations, mais une fois que le classement des poèmes du Shinkokin sera achevé, alors oui je vous écouterai ", ce qui me parut une manière bien élégante de répondre. Nous avions saisi le ton légèrement ironique des propos de Gotoba sans pour autant réagir ${ }^{43}$.

La compilation de l'anthologie était un travail de chaque instant qui ne permettait plus à Gotoba de s'occuper des affaires courantes de l'État. Les poèmes affluaient de toutes parts, envoyés par des gens qui souhaitaient - honneur suprême! - voir une de leurs œuvres retenue dans le Shinkokin waka shû. Cette machine infernale faisait feu de tout bois; elle réclamait sans cesse de nouveaux poèmes qui puissent s'insérer parfaitement dans l'ensemble, rehausser un motif ou combler un interstice laissé vacant. La moindre manifestation poétique voyait ses produits immédiatement évalués et, le cas échéant, recyclés. Ainsi :

Comme le travail de classification touchait à sa fin, les membres du Bureau de la poésie ressentirent la tristesse d'avoir bientôt à se séparer, aussi organisèrent-ils un concours de poèmes, les anciens rivalisant contre les jeunes ${ }^{44}$. L'Empereur retiré fut chargé de déterminer quels étaient les poèmes gagnants et les perdants. C'est à cette occasion qu'Ietaka composa son poème « le daim solitaire et mouillé » qui fut par la suite inclus dans l'anthologie ${ }^{45}$. D'une manière générale, pour cette anthologie, on n'a pas systématiquement rejeté les poèmes qui comportaient des 
fautes, la priorité était la qualité d'ensemble du poème. En outre, le principe [de cette anthologie] était de revisiter les poèmes anciens ${ }^{46}$.

Dès le choix du titre Shinkokin waka shû / Nouveau recueil de poèmes anciens et modernes, Gotoba montra sans ambiguïté qu'il concevait son anthologie comme une relecture du Kokin waka shû / Recueil de poèmes anciens et modernes, la première anthologie impériale. Il ne s'agissait toutefois pas d'une simple imitation; le prestigieux modèle devait être surpassé. Alors que les anthologies impériales précédentes rassemblaient les poèmes que le ou les compilateurs jugeaient les meilleurs, Gotoba décida quant à lui de privilégier le soin avec lequel les poésies s'intégraient en un tout parfaitement cohérent et homogène, faisant en cela œuvre de pionnier. La nécessité de préserver au sein des principales sections la progression du thème et la qualité d'ensemble impliquait que l'on retînt également des poèmes qui, pris individuellement, pouvaient sembler médiocres ou même comporter des imperfections, mais qui, intégrés dans une séquence, se fondaient parfaitement, permettant ainsi de rehausser la beauté d'une pièce ou d'un motif. Ce travail de classification présentait parfois, comme nous le montre la citation suivante des notes journalières de Teika, des problèmes qui dépassaient le simple cadre esthétique:

Genkyâ 2 (1205), II, 22: Vers dix heures du matin, je me rends au Bureau de la poésie. Munenobu, Ienaga, Tomochika et Hideyoshi s'y trouvent déjà, coupant et collant [les poèmes de l'anthologie]. Son Excellence [Yoshitsune] vient également; écartant les parois mobiles, il nous observe [...]. Tomochika présente alors une boîte repas compartimentée (ornée d'un élégant motif de livres). Le vin est présenté dans un tube semblable à un manuscrit roulé. Je me retire au crépuscule. Aujourd'hui, nous avons achevé la section des poèmes d'amour ainsi que la section consacrée aux poèmes bouddhiques (shakkyôka). Comme les poèmes sur des sujets divers (zô) sont trop nombreux, nous décidons d'attendre que l'assemblée soit plus fournie [pour nous en occuper]. Nous nous attelons aux poèmes relatifs aux divinités shintô (jingi). Je dis alors à Ienaga que je crains de m'occuper de cette section de l'anthologie, étant encore en période de deuil [et donc impur]. Les poèmes [attribués à] des dieux (kami no uta) sont extrêmement nombreux. Il est en outre difficile de déterminer dans quel ordre les classer. Bien que nous puissions nous tenir aux principes de classement suivis jusqu'alors, nous devons avant tout nous procurer les anthologies précédentes [pour savoir comment ces poèmes y sont arrangés] ${ }^{47}$. En mon for intérieur, je me dis qu'aligner les noms de divinités pourrait se révéler extrêmement dangereux, je ne participe donc pas au classement.

Teika, qui porte encore le deuil de son père, exprime ici sa réticence à participer au classement des poèmes attribués aux divinités Shintô. Alors que 
la mise au point de la section consacrée aux poèmes bouddhiques ne semble pas avoir soulevé la moindre objection de la part des compilateurs, le fait même d'avoir à procéder à un classement de ces poèmes fait craindre à Teika une possible malédiction dans le cas où une divinité ne serait pas satisfaite de la place attribuée à son poème ${ }^{48}$. Il est intéressant de noter que dans l'anthologie, les poèmes shintô sont tout naturellement placés avant les poèmes bouddhiques. Si l'on examine de plus près le classement opéré par les compilateurs pour cette section, nous remarquons que les poèmes attribués à des dieux (poèmes «révélés» ou «manifestés» à une tierce personne), figurent en tête de la section; viennent ensuite des poèmes composés lors des banquets donnés à l'issue de leçons sur le Nihon-shoki / Chroniques du Japon (nous reviendrons bientôt plus en détail sur ces manifestations), et enfin les poèmes composés à l'occasion de pèlerinages à des sanctuaires, lors de célébrations religieuses ou encore des œuvres composées sur des thèmes imposant la mention d'une divinité. Gotoba recommanda en outre que les poèmes composés par des dieux soient placés dans l'ordre des saisons ${ }^{49}$, ce qui fut fait ${ }^{50}$.

En plus de leur travail de compilation, les membres du Bureau de la poésie devaient à tour de rôle gratifier leurs compagnons d'un repas. Teika décrit en détail dans ses notes journalières le régal qu'il offrit le 12 du deuxième mois. Nous noterons l'extrême raffinement de la disposition des mets par laquelle Teika cherchait à évoquer l'une des œuvres poétiques les plus importantes de l'époque de Heian, l'Ise monogatari ou Contes d'Ise ${ }^{51}$ :

Genkyû 2 (1205), II, $23:[\ldots]$ Vers midi Ienaga me dit [...] qu'il faut me rendre au Bureau de la poésie; Ariie, Ietaka et Munenobu, entre autres, s'y trouvent déjà, coupant et collant [les poèmes de l'anthologie]. [...] Pendant ce temps je prépare de la nourriture que je porte et installe [au Bureau de la poésie]. Quand Ienaga, Kiyonori et les autres reviennent et voient ce que j'ai apporté, ils me donnent quelques conseils sur la manière de présenter les mets et me recommandent de ne pas y toucher car cela mérite que l'Empereur retiré le voie. Je ne réponds rien et dispose les différents éléments sans détruire [l'harmonie d'ensemble]. Dans une grande boîte rectangulaire les mets et les boissons [étaient disposés comme suit]: sur un fin plateau de bois, j'ai posé un plat creux en terre cuite; j'en tapissai le fond de feuilles de chêne et sur ces dernières disposai un petit tas d'algues comestibles que je recouvris d'autres feuilles. Sur celles-ci j'écrivis le poème:

$\begin{array}{ll}\text { Watatsumi no } & \text { Les algues } \\ \text { kazashi ni sasu to } & \text { Que le dieu des mers pour orner sa chevelure } \\ \text { iwahu momo } & \text { Garde précieusement }{ }^{52} \text {. }\end{array}$

Sur un autre plateau j'ai peint un motif et posé dessus une coupe. J'ai enveloppé de papier rouge le col d'un flacon à alcool que j'ai en réalité rempli de bouillon de 
poule. Dans une boîte à repas tripode [et à plateaux superposés] j'ai disposé des fleurs d'oranger et sur le papier [décoratif destiné à recevoir les aliments] j'ai écrit à l'aide d'un petit bâton le poème «Il a la même fragrance / Que la manche de mon ancien amant " ${ }^{53}$. Les fleurs d'oranger, je les ai placées sur le plateau supérieur; en dessous, j'ai aménagé trois niveaux que j'ai compartimentés et sur lesquels j'ai disposé six variétés de gâteaux. Dans une autre boîte repas, j'ai placé des algues comestibles et écrit le poème «Même si pour tapis / Nous n'avions que nos manches ${ }^{54}$ et sur les plateaux inférieurs j'ai placé, comme pour les gâteaux, six variétés de poissons et de volailles. J'ai orné un flacon vert - dont je n'avais pas enveloppé le col - d'une fleur de glycine (en la maintenant à l'aide de fil car la grappe était particulièrement grande) et l'ai rempli d'alcool. J'ai roulé ensuite, comme on le fait pour certaines lettres, des feuilles de papier épais (avec motifs en inclusion) et j'y ai glissé les baguettes. Sur l'enveloppe j'ai écrit «Étriers de Musashi » 55 . Dans une boîte repas, j'ai mis du riz, sur celui-ci j'ai empilé des gâteaux de riz pilé, de façon à le cacher entièrement. Voilà ce que j'ai fait préparer. En dehors de cela, j'ai apporté discrètement des coupes pour servir le vin, les ai posées dans un endroit calme et ne les ai pas sorties. Tout ceci fait allusion aux Contes d'Ise. À la nuit tombée, nous avons achevé les corrections du premier volume de poèmes divers $(z \hat{o})$, puis chacun s'est retiré.

Malgré la lassitude manifeste des compilateurs, et leur mauvaise humeur, le travail se poursuit, imperturbablement:

Genkyâ 2 (1205), II, $26:[\ldots]$ Vers dix heures du matin, je me rends au Bureau de la poésie. Ietaka ason ne vient pas. On continue de corriger le dernier livre de poèmes divers. Aujourd'hui, on corrige également un peu les livres I et II des poèmes d'amour. Les gens sont toujours de mauvaise humeur. La section «amour» de l'anthologie doit être extrêmement élégante; il subsiste encore de nombreux défauts, aussi, oubliant notre réticence, nous mettons-nous à corriger jusqu'à la tombée de la nuit $[\ldots]$.

Gotoba voulait que son anthologie reflète la vivacité de la poésie de son temps. Aussi demanda-t-il aux compilateurs de remplacer le premier poème de certains livres - dont deux livres d' amour - par une œuvre de Teika, Ietaka et la fille de Monseigneur Shunzei, trois fleurons de son cercle poétique ${ }^{56}$ :

Genkyû 2 (1205), III, 2: [...] Comme l'Empereur retiré nous dit: «Vous avez, en règle générale, placé en tête de chaque livre un poème composé par un auteur qui n'est plus de ce monde, cela ne va pas. Il faut placer au début de chaque volume un poème des trois auteurs suivants: Teika, Ietaka et la fille de Monseigneur Shunzei », nous avons effectué les corrections. Un poème d'Ietaka fut placé en tête du volume II des poèmes d'automne, une œuvre de la poétesse en tête du deuxième volume de poèmes d'amour et enfin, un de mes poèmes au début du livre $\mathrm{V}$ des poèmes d'amour. De mon propre chef, je les aurais placés en fin de volume. Je trouve cette remarque de l'empereur très intéressante. 
Dans la mise au point de l'anthologie rien ne devait être laissé au hasard. Les compilateurs devaient savoir combien de poèmes avaient été sélectionnés pour chaque poète et quels poètes avaient été retenus. Aussi, en Genkyû 2 (1205), le 4 du troisième mois, Teika établit-il l'index des poètes contemporains dont les œuvres ont été retenues et le lendemain, le 5, celui des poètes défunts. Malgré le travail fourni et les indications dont il dispose, Teika peine encore à se faire une idée précise de ce que sera l'anthologie achevée:

Genkyû 2 (1205), III, $25:[\ldots]$ Je me rends au Bureau de la poésie où je travaille jusqu'à la fin de la journée. En début de soirée, je me retire. Je me sens particulièrement mal à l'aise. Je n'ai pas encore pu voir cette anthologie [complète]. Elle contient sans doute encore beaucoup d'erreurs. Le trop grand nombre de participants gêne la compilation.

Malgré l'état d'inachèvement de l'anthologie, Gotoba tenait à tout prix à célébrer la fin de la compilation. Cette idée lui était sans doute venue quelques semaines auparavant, au moment de la mise au point de la section consacrée aux poèmes shintô, quand les compilateurs avaient classé des waka composés lors de banquets donnés à l'issue de leçons sur le Nihonshoki, la première des «Six Histoires» du Japon, achevée en $720 .{ }^{57}$ Les leçons sur le Nihonshoki furent données à la Cour pour la première fois en 811 (ou 812), puis à peu près tous les trente ans jusqu'en 965 . Ces leçons s'achevaient en général par un banquet à l'issue duquel on composait des poèmes ayant pour thème les personnages - dont les divinités - apparaissant dans l'ouvrage ${ }^{58}$. Gotoba décida de s'inspirer de ce type de manifestation pour son propre banquet. Il fallut alors presser les différents acteurs pour qu'ils produisent à temps une copie de l'anthologie et des préfaces. Le 22 du troisième mois Gotoba demanda à Yoshitsune s'il lui était possible de préparer une copie de l'anthologie pour le 27 et de lui présenter avant cette date la préface en japonais dont il avait la charge ainsi que des sujets qui pourraient être distribués pour les compositions poétiques qui suivraient le banquet ${ }^{59}$. Yoshitsune fit savoir qu'il lui serait difficile de s'acquitter de tout cela dans les temps. Teika retrouva cependant Ietaka au Bureau de la poésie où ils recopièrent en entier l'anthologie et en corrigèrent les coquilles sur la moitié ${ }^{60}$. Le lendemain, on fit savoir que la date du banquet était arrêtée au 26 et qu'en l'état des choses on se contenterait pour la cérémonie d'une copie provisoire de l'anthologie; la mise au propre serait faite après le banquet et la préface en japonais rajoutée ultérieurement. Fujiwara no Chikatsune, l'un des plus grands savants confucianistes de ce temps, avait été chargé par Gotoba, le 22 du septième mois de l'année 1204 (Genkyû 1), de rédiger, en nom et place de l'empereur retiré, la préface en chinois; le 21 du deuxième mois de 
l'année suivante (1205), il vint l'apporter au Bureau de la poésie. Après l'avoir examinée Gotoba demanda à Yoshitsune d'y apporter quelques corrections $^{61}$. On disposerait donc au moins de la préface en chinois pour la cérémonie.

Il n'existait aucun précédent de célébration pour la fin de la compilation d'une anthologie impériale. Comme nous l'avons vu, Gotoba s'inspirait d'une cérémonie qui était sans rapport avec la situation présente: Teika refusa donc d'y prendre part. Il est intéressant de noter qu'lenaga, qui naturellement assistait au banquet, consacre l'essentiel de sa narration au fait que le pauvre Kinsada qui tenait le luth pendant le divertissement musical joua faux du début à la fin, et que la nombreuse assistance dut en faire des gorges chaudes ${ }^{62}$. Teika, qui n'y avait pas pris part, consigne avec une extraordinaire minutie le déroulement de la cérémonie tel que Yoshitsune le lui rapporta le lendemain:

Genkyû 2 (1205), III, 27: [...] La célébration se déroule dans le pavillon Hirogosho (au nord du Bureau de la poésie). L'Empereur retiré siège au nord [de la salle, séparé de l'assistance par] deux paravents placés d'est en ouest. Au sud de ceux-ci, deux autres paravents ont été placés parallèlement (en position nord-sud) pour que les hauts dignitaires puissent s'asseoir en deux rangées, face-à-face [dos aux paravents]. Au nord-ouest, des stores ajourés font une séparation; [derrière ceux-ci] Inpumon-in ${ }^{63}$ et le prince héritier ${ }^{64}$ assistent [à la célébration]. Son excellence [Yoshitsune] et l'ancien ministre des affaires suprêmes [Yorizane] (habillés du costume de Cour et du bonnet) prennent place les premiers, puis, après un bon moment, [l'Empereur retiré] fait son entrée. Ienaga s'assied au bord de l'endroit où se trouve [Gotoba-in] et, à la demande du monarque, invite les hauts dignitaires [à prendre place]. [Michiteru] de la garde des portes, section de gauche, et [Michitomo] de la garde des portes, section de droite, de même que Takahira et Tsuneie (en costume de Cour) pénètrent dans la salle et s'installent. Yorizane, à la demande de l'Empereur retiré, appelle Ienaga et Ariie. Ariie prend place devant la table - la table et le grand chandelier au pied découpé avaient été disposés préalablement. Sur la table [un manuscrit] du Shinkokin shû est posé; Ariie lit la préface. Monseigneur Michitomo, venu le seconder dans sa tâche, poursuit la lecture. Après avoir lu les quatre ou cinq premiers poèmes de la section de printemps, les lecteurs se retirent. Ensuite, chacun des poètes de l'assistance présente un poème, à commencer par l'adjoint de la garde des gendarmes Tomochika, suivi de Hideyoshi, Kiyonori, Ienaga (qui fait déposer son poème sur la table par un serviteur), du général en troisième Tadasada, de l'adjoint mineur du département du palais Munenobu [...]. Son excellence [Yoshitsune], sans se lever de son siège, fait déposer son poème. Ensuite, on appelle Ietaka pour qu'il officie comme lecteur. À la demande de l'Empereur retiré, Ariie se place derrière le lecteur et déclame les poèmes. Puis, les poètes se retirent. (Dans les deux cas l'ancien ministre des affaires suprêmes Yorizane a servi de lecteur.) Ensuite, les 
musiciens prennent place; des courtisans du cinquième rang apportent les instruments. Takanaka est aux cliquettes, le grand contrôleur de droite [Kinsada] au luth, Tsunemichi à la cithare, Takamasa au koto, Monseigneur Chikakane à la flûte, Morikane au hautbois et Takahira à l'orgue à bouche. À la fin du divertissement [Gotoba-in se retire], puis les participants s'en vont [...]. Ceci étant dit, quelle raison y a-t-il de donner pareille célébration? Il n'existe pas de précédent à cela. Organisée dans la précipitation, rien n'était au point; les poètes [présents] n'étaient pas [tous] de réels poètes. Le choix qui fut fait est douteux.

La célébration ne marquait aucunement la fin de la compilation. Sitôt la cérémonie achevée, les travaux de mise au point de l'anthologie se poursuivent. Teika écrit dans ses notes journalières:

Genkyú 2 (1205), III, $28:[\ldots]$ Ienaga me fait dire qu'il faut que je me rende au Bureau de la poésie: je m'exécute avec empressement. Il arrive en portant les poèmes composés lors du banquet [célébrant] la fin de la compilation. Nous les copions. L'Empereur retiré nous fait dire qu'il faut que nous examinions l'anthologie. Nous jetons donc un coup d'œil. Dans le volume de poèmes de félicitations, les œuvres de Kiyotada et Tsunenobu sur le premier jour du rat sont [trop] semblables ${ }^{65}$ : $j$ 'insère donc plusieurs poèmes entre eux. De même, parmi les poèmes de doléance, deux œuvres que j'avais sélectionnées [...] étaient [trop] similaires. Quand j'expose le problème de ces deux [paires de] poèmes à l'Empereur retiré, il me dit de laisser le poème de Tsunenobu et de ne pas enlever les deux poèmes de doléance, de les laisser à côté l'un de l'autre.

Tandis que Teika et d'autres membres du Bureau de la poésie travaillent à la mise au point de l'anthologie, Yoshitsune achève la préface en japonais. Dans ses notes journalières, Teika nous dit qu'ayant pu la lire, il la trouve "particulièrement remarquable»; il recommande en outre à Yoshitsune de la présenter le plus rapidement possible à Gotoba ${ }^{66}$. Entre le 28 du troisième mois de l'année Genkyû 2 (1205) et le neuvième mois de l'année Jôgen 4 (1210), plusieurs révisions de l'anthologic furent faites qui devaient avoir pour résultat le rajout d'une soixantaine de nouveaux poèmes. Ceux-ci furent pour la plupart choisis parmi les œuvres composées lors de manifestations poétiques organisées dans le but de produire de nouvelles pièces pouvant être intégrées dans l'anthologie. L'intégration de nouveaux poèmes impliquait parfois la suppression de waka qui avaient préalablement été sélectionnés, ce qui, quand l'auteur était de ce monde, provoquait, nous dit Ienaga, un certain émoi :

Ainsi, même si le banquet avait déjà eu lieu, [l'Empereur retiré] ne semblait pas pressé d'obtenir une copie définitive [de l'anthologie]. On continua par la suite à rajouter et retrancher des poèmes et on inclut même des œuvres composées lors d'un concours de poèmes chinois et japonais [organisé après le banquet] ${ }^{67}$ Ceux 
dont on rejetait des poèmes qui avaient été préalablement sélectionnés se plaignaient amèrement, et les entendre faisait naître en moi un sentiment de culpabilité. Ils n'avaient à aucun moment pensé [que leurs poèmes pussent être écartés] ; après avoir cru à leur sélection, apprendre leur rejet les avait abattus. Leur mine défaite faisait tant de peine à voir que même les gens qui n'étaient pas directement concernés versaient des larmes ${ }^{68}$. Tant que la mise au point définitive n'avait pas eu lieu [les poètes écartés] ne se résignaient pas et adressaient leurs suppliques [aux dieux et aux bouddhas] sans en oublier aucun. J'étais ému d'entendre à quel point leur sentiment était profond. Il m'a semblé fastidieux de noter les poèmes produits cette nuit-là [c.-à-d. au cours du banquet], aussi me suisje abstenu ${ }^{69}$.

En Ken.ei 1 (1206), le 7 du troisième mois, le brillant Yoshitsune s'éteignit brutalement à l'âge de trente-sept ans. Figure centrale dans l'entreprise de compilation de l'anthologie, c'est lui qui avait été chargé, entre autres, d'en rédiger, au nom de l'empereur, la préface en japonais. Les notes journalières de Teika sont silencieuses durant les quatre premiers mois de cette année, nous ne pouvons donc les citer ${ }^{70}$. Il n'en demeure pas moins que son chagrin dut être extrêmement profond. Teika, qui était au service de Yoshitsune, perdait son protecteur et son meilleur soutien, il perdait aussi un complice ses notes journalières nous le prouvent ${ }^{71}$. Le 19 du sixième mois, Teika fut chargé d'examiner des points problématiques relevés par Yoshitsune dans l'anthologie:

Temps dégagé. Vers trois heures, tonnerre et pluie violente qui ont cessé le soir. L'Empereur retiré me fait savoir par Kiyonori qu'il reste [des problèmes à régler] concernant le nombre de poèmes du Shinkokin, je m'empresse donc de me rendre au palais. Le chef du département du trésor Fujiwara no Ariie fut également appelé, mais il n'est pas venu. Après les violents coups de tonnerre, Kiyonori vient. Il me remet cinq volumes du Shinkokin et me dit: «Dans ces volumes sont insérées des feuilles de papier où le regretté Yoshitsune a noté quelques remarques; veuillez les examiner.» Dans la section des poèmes de félicitations, une pièce attribuée au ministre de droite Fujiwara no Morofusa figurait comme ayant été composée lors du banquet suivant le Concours de poèmes chez la princesse Ippon Ryôshi; [Yoshitsune] avait inséré à cet endroit un morceau de papier sur lequel il avait noté ses doutes à ce sujet [et en effet] le concours [n'était pas celui qui figurait sur le manuscrit] mais bien le Concours de poèmes chez la princesse Yûshi. (Ce poème a été sélectionné par Minamoto [no Michitomo] qui s'est trompé à la fois d'époque et de personne ${ }^{72}$.) Dans la section des poèmes sur l'affliction, on trouvait une pièce - également sélectionnée par Michitomo attribuée à l'ancien ministre des affaires suprêmes entré en religion Chisoku-in qui, selon la notice, «l'avait envoyé chez dame Shinshôshô au moment où mourut [son épouse] la Dame du Second Rang ${ }^{73} \gg$. [Yoshitsune doutait de l'exactitude du] Second Rang [et en effet, elle était Dame du Premier Rang Mineur]; tout cela est 
d'une telle négligence! Il m'est difficile de concevoir que l'on puisse ignorer de telles choses. Je suggère de demander au secrétaire des affaires suprêmes de quelle façon régler ces points $[\ldots]$.

La compilation du Shinkokin waka shû, à laquelle Yoshitsune était si étroitement associé, semble être devenue depuis sa mort un fardeau encore plus insupportable. Teika écrit dans ses notes journalières:

Jôgen 1 (1207), II, 26: [...] Je me rends au palais [...]; au Bureau de la poésie il est encore question du Shinkokin (cela semble ne devoir jamais finir!) [...].

Et neuf mois plus tard:

Jôgen 1, XI, 8: [...] Je me rends au palais. À la demande de l'Empereur retiré, je corrige encore le Shinkokin (les corrections changent sans arrêt). Mon seul rôle consiste à corriger, ce qui pour moi n'a aucun intérêt [...].

Les corrections se poursuivront ainsi jusqu'au neuvième mois de Jôgen 4 (1210), période à laquelle furent inclus dans l'anthologie treize nouveaux poèmes composés pour une manifestation organisée par Gotoba ${ }^{74}$. Après cette date, Teika ne mentionne plus aucune séance de corrections; l'anthologie devait donc être, à quelques détails près, achevée. Ce n'est toutefois que six ans plus tard, en Kenpô 4 (1216), le vingt-six du douzième mois, qu' lenaga, en tant que secrétaire du Bureau de la poésie, acheva la copie - définitive - de l'anthologie. C'est sur ce manuscrit, dont l'original n'a malheureusement pas été conservé, que se fondent toutes les éditions de notre texte. Il aura donc fallu quinze années d'un travail souvent harassant pour produire la huitième anthologie impériale. Elle contient deux préfaces (l'une en chinois, l'autre en japonais) et près de deux mille waka ${ }^{75}$. Aucun ordonnateur avant Gotoba ne s'était autant impliqué dans le travail de compilation. L'empereur retiré devait tout contrôler, tout savoir. C'est ainsi que Teika eut à établir les index des poètes vivants et morts dont les nuvres avaient été retenues; ceux-ci devaient permettre de savoir quels étaient les poètes les mieux représentés et d'équilibrer l'ensemble. Aucun détail n'était laissé au hasard, chaque poète figurait dans l'anthologie selon son prestige. Le poète le mieux représenté est Saigyô (1118-1190) avec quatre-vingt-quatorze waka retenus. Saigyô, qui n'était plus de ce monde lorsque fut ordonnée l'anthologie, est considéré comme le plus grand poète de ce temps; sa prééminence est donc, aux yeux des compilateurs - et des Japonais contemporains - tout à fait justifiée. Vient ensuite Jien (1155-1225), avec quatre-vingt-douze poèmes. Jien, qui était le supérieur général de l'école bouddhique Tendai, appartenait à la puissante famille Kujô. Il était membre du Bureau de la poésie et considéré comme excellent poète. Son neveu, le brillant Yoshitsune, personnage central dans le 
travail de compilation, le suit avec soixante-dix-neuf poèmes retenus. Shunzei, le père de Teika, est représenté par soixante-douze poèmes et Teika par quarante-six. Ietaka, membre du Bureau de la poésie et également compilateur, compte quarante-trois poèmes retenus. La princesse impériale Shokushi Naishinnô, poétesse de génie qui fut un temps l'élève de Shunzei, est la femme dont le plus grand nombre de waka - quarante-neuf - ont été sélectionnés. Gotoba, le commanditaire, compte trente-quatre poèmes dans le recueil, ce qui est un fait sans précédent. À titre de comparaison, rappelons qu'aucun poème de l'empereur Daigo ne figure dans la première anthologie impériale, le modèle de la nôtre. Ce fait apparemment anodin est, dans la subtile mécanique de la compilation, extrêmement significatif: Gotoba se place à la suite des meilleurs poètes de son temps au centre de son grand œuvre. Aucun empereur n'avait avant lui joué un rôle aussi important dans la compilation d'une anthologie. Celle-ci était pour lui, bien plus qu'une œuvre d'art, le superbe symbole de sa puissance. Rappelons que les préfaces ont été rédigées en son nom, c'est donc sa vision du monde - et de l'ordre de celuici - que, par le truchement de la poésie, l'anthologie devait exprimer. Elle devait donc être plus importante, imposante, parfaite, que toutes les anthologies impériales précédentes. Dans son étude intitulée Les Anthologies en France, Emmanuel Fraisse souligne qu' «en tant qu'objet fondateur d'une identité [...] l'anthologie s'est vue très tôt conférer un rôle littéraire mais aussi idéologique ${ }^{76} »$; nous avons pu mesurer au cours de notre enquête l'importance de cette dimension idéologique - dans le choix des poètes et la place conférée à leurs poèmes, mais aussi dans l'esthétique générale de l'œuvre - qui sous-tend le travail de compilation d'une anthologie impériale telle que le Shinkokin waka shû.

Le cinquième mois de l'année Jôkyû 3 (1221), Gotoba tenta par un coup d'État de renverser le gouvernement militaire qui, depuis 1185 , dominait les provinces de l'Est, espérant ainsi rétablir son pouvoir sur l'ensemble du territoire. Cependant, après un mois de campagne appelé «troubles de Jôkyû », son parti fut vaincu. Il fut alors exilé à Oki, archipel de petites îles battues par les vagues de la mer du Japon, réputées pour la rigueur de leur climat. Il emporta dans son exil le Shinkokin waka shû, auquel il travailla encore de 1234 à 1236 environ, retranchant quelque quatre cents poèmes et ajoutant une postface dans laquelle il précise que cette dernière version, appelée aujourd'hui «manuscrit d'Oki», Oki-bon, doit être considérée comme l'état définitif de son anthologie. C'est toutefois le manuscrit copié par Ienaga, achevé en 1216, qui fut et reste la version officielle du Shinkokin waka shâ. Au début de l'année 1239 Gotoba mourut, toujours en exil. Il n'avait pas soixante ans. Le lenaga nikki, qui avait été achevé avant le coup 
d'État et l'exil de Gotoba, ne mentionne pas ces derniers avatars de l'anthologie. Teika, dont les relations avec l'empereur s'étaient considérablement dégradées au fil des années, n'eut, semble-t-il, plus aucun contact avec Gotoba pendant les dix-neuf années que dura son exil. La page du Shinkokin waka shû était définitivement tournée et les leçons tirées : quand en 1232 Teika reçut la commande de la neuvième anthologie impériale, il n'eut qu'une exigence, être nommé seul compilateur, ce qui lui fut accordé.

\section{Notes}

1. Gotoba pratiquait la balle au pied, kemari, et une sorte de hockey sur gazon appelé gichô.

2. Le premier exemple d'anthologie impériale est le Kokin waka shû, « Recueil de poèmes anciens et modernes", commandée par l'empereur Daigo en 905 et achevée vers 913. Il servira de modèle - plus ou moins scrupuleusement respecté - aux vingt anthologies impériales compilées par la suite. Notre anthologie est la huitième.

3. Certaines anthologies impériales contiennent également quelques chôka, «poèmes longs » (la première, le Kokin waka shû, en contient cinq contre plus de mille waka). Notre anthologie ne contient quant à elle que des waka.

4. Nous utilisons pour ce texte l'édition annotée par Ishida Yoshisada et Satsukawa Shûji, lenaga nikki zenchûkai, Yûseidô, 1968 (abrégée dorénavant en $I N$ ). Nous avons également, pour certains passages - précisés dans les notes -, utilisé l'ouvrage collectif, lenaga nikki, kôhon, kenkyû, sôsakuin, Kazama shobô, 1985.

5. Pour ce texte, nous utilisons l'édition en 3 volumes, Kokusho Kankôkai, 1969 (désignée dorénavant par les initiales $M G K$ ). Signalons que pour identifier les passages du $M G K$, nous donnons d'abord l'année selon les ères japonaises, puis, entre parenthèses, l'équivalent dans notre calendrier, suivi du mois en chiffres romains et enfin du jour en chiffres arabes. Par exemple: Kennin 1 (1201), VII, 5 , signifie: cinquième jour du septième mois de la première année de Kennin (1201).

6. Indications données par le Waka daijiten, Meijishoin, 1986, article Ienaga nikki.

7. Les Mémoires relatent des événements qui eurent lieu en 1197 pour les plus anciens et en 1207 pour les plus récents.

8. On pourra lire l'ouvrage que nous avons consacré à ce poète majeur: Fujiwara no Teika (1162-1241) et la notion d'excellence en poésie. Théorie et pratique de la composition dans le Japon classique, Collège de France / Institut des Hautes Études Japonaises, 2001.

9. Teika commença la rédaction de ses notes journalières en 1180 - il avait alors 18 ans - et les poursuivit jusqu'en 1235. Sur les notes journalières que tenaient en chinois certains personnages, voir Francine Hérail, Notes journalières de Fujiwara no Michinaga, ministre à la cour de Hei.an (995-1018), vol.1, Librairie Droz, 1987, p. 26-37. 
10. Il ne faut pas oublier que les notes journalières servaient également de recueils de précédents pour les générations futures qui devaient pouvoir y trouver quelle attitude adopter dans une situation particulière. La précision était donc une qualité indispensable à ce genre d'ouvrages. L'extrême minutie de certains passages du Meigetsuki, en particulier les descriptions de célébrations officielles et du protocole, ne sont pas sans rappeler les Mémoires de Saint-Simon.

11. MGK, Kennin 1 (1201), VII, 27.

12. C'est-à-dire les personnes autorisées à pénétrer dans la «salle d'en haut» du Shishinden, la résidence de l'empereur.

13. En Kennin 1 (1201) Yoshitsune n'était pas encore régent ministre de gauche; il n'obtiendra ce titre que l'année suivante (IN, p. 55).

14. Ienaga ne note que la moitié du prénom qui est complété par les commentateurs.

15. Ason est un titre honorifique

16. Shakua est le nom religieux de Fujiwara no Shunzei (1114-1204), le père de Teika.

17. Teika nous dit (MGK, Kennin 1 [1201], VIII, 5) que la nomination d'Ienaga au poste de secrétaire du Bureau de la poésie se fit collectivement.

18. Dans ce poème qui loue l'intérêt que Gotoba porte à la poésie, et la profusion de compositions qu'il devrait susciter, Ienaga recourt aux images associées à la mer: les algues, les vagues et la Baie de Waka. Les algues mentionnées étaient grillées pour en extraire le sel (sur ce procédé d'extraction, voir Bernard Frank, Annuaire de l'École Pratique des Hautes Études, IV section, Sciences historiques et philologiques 1976-1977, p. 1042); l'un des sens de waka étant «poésie », Ienaga utilise ce toponyme pour évoquer une Baie de la Poésie où les poèmes seraient aussi nombreux que des vagues. Précisons que la Baie de Waka, Waka no ura, se situait dans l'ancienne province de Kii (actuel département de Wakayama).

19. IN, p. 52-53.

20. Voir, MGK, Kennin 1 (1201), VII, 26.

21. Gotoba semble avoir accordé une grande importance à «l'obligation de présence» des membres du Bureau de la poésie. Ainsi, nous dit lenaga, en Kennin 1 (1201), VIII, 15, il y eut le soir une séance de compositions au Bureau de la poésie. Tomochika, qui ne souhaitait pas y participer, s'éclipsa discrètement. Gotoba s'en aperçut et, mécontent, le convoqua le lendemain matin, l'obligeant à passer toute la journée enfermé au Bureau de la poésie. Il ne le libéra que le 17 (IN p. 59-61).

22. La manifestation poétique dont il est ici question n'a pu être identifiée.

23. IN, p. 58.

24. Nous ne pouvons, faute de place, évoquer les différentes manifestations poétiques organisées par Gotoba; le lecteur intéressé peut se reporter à l'ouvrage de Robert N. Huey qui vient de paraître, The Making of Shinkokinshû, Harvard University Asia Center, Harvard East Asian Monographs 208, 2002, ou bien encore à notre ouvrage, op. cit., p. 35-50 en particulier. Signalons que les deux ouvrages japonais de référence sur ces questions sont: Ariyoshi Tamotsu, 
Shinkokin waka shû no kenkyû, kiban to kôsei, Sanseidô, 1968 et Gotô Shigeo, Shinkokin waka shû no kisoteki kenkyû, Hanawa shobô, 1968.

25. IN, p. 72-73.

26. Voir également le récit que fait Ienaga de la mort de Jakuren, $I N$, p. 75-76.

27. Pour ce passage, nous suivons l'édition et le commentaire qui en est fait dans Meigetsuki kenkyû, $\mathrm{n}^{\circ}$ 1, novembre 1996, p. 8.

28. MGK, Kennin 3 (1203), IV, 11.

29. MGK, Kennin 3 (1203), IV, 20.

30. Voir la description de cette célébration dans $I N, 122-147$.

31. Voir le $M G K$, Genkyû 1 (1204), XI, 26-30, pour une description de la maladie et de la mort de Shunzei. La mort de Shunzei est également décrite dans IN, p. 149150.

32. Les principales différences sont les sections «poèmes de séparation », ribetsu et "poèmes [incluant de façon cryptée] des noms de choses» mono no na qui ne figurent pas dans notre anthologie. Les deux sections «Shintô», jingika et «Bouddhisme» shakkyôka, quant à elles, n'apparaissaient pas dans la première anthologie impériale.

33. Sur les principes d'intégration de la poésie japonaise classique dans les anthologies impériales, voir l'article de Konishi Jin'ichi, Brower Robert H., Miner Earl, «Association and progression: Principles of Integration in Anthologies and Sequences in Japanese Court Poetry, A.D. 900-1350 ", Harvard Journal of A siatic Studies, vol. 21 (déc., 1958), p. 67-127.

34. Dans ses notes journalières, Teika désigne souvent les personnages par leur seule fonction de cour. Ainsi trouve-t-on à cet endroit l'«intendant du bureau de police» qu'Imagawa Fumio dans son Meigetsuki shô désigne comme étant Michitomo. Pour Robert Huey, op. cit, p. 268, ce titre désignerait en fait Fujiwara no Hideyoshi. Or, si l'on en croit le Waka daijiten, Hideyoshi ne fut associé au Bureau de la poésie qu'en 1205.

35. Les deux premiers de l'anthologie.

36. Le Tanabata est une fête qui célèbre la rencontre annuelle - le sept du septième mois - de l'étoile du Bouvier avec celle de la Tisserande. Les poèmes de Tanabata ( $n^{\circ} 313$ à 327$)$ sont classés dans la section «automne » de l'anthologie.

37. Gotoba prenait, là encore, modèle sur la première anthologie impériale. Les six anthologies impériales qui précèdent la nôtre sont soit dépourvues de préface, soit dotées de la seule préface en japonais.

38. Le banquet eut lieu en réalité le 26 du troisième mois. Voir $I N$, p. 212.

39. Le manuscrit est corrompu à cet endroit; nous suivons pour ce passage les indications des commentateurs du $I N$ (p. 212).

40. La préface en chinois fut en fait achevée juste à temps pour la cérémonie.

41. Là encore Ienaga se trompe. Comme nous l'avons vu, le travail de classification a commencé le septième mois.

42. IN, p. 211

43. $I N$, p. $215-216$. 
44. On ne sait pas à quelle date précise eut lieu ce concours de poèmes. Un passage des notes journalières de Teika (Genkyû 2 (1205), III, 2) nous apprend qu'à cette date le poème d'letaka était déjà composé : le concours eut donc lieu avant.

45. Il s'agit du poème $n^{\circ} 437$, placé en tête du deuxième livre d'automne et composé sur le sujet «daim au crépuscule»:

Shita momiji

katsu chiru yama no

yuhu shigure

nurete ya hitori

shika no nakuran
Dans la montagne

Couverte de feuilles pourpres tombées

Une ondée vespérale -

Le daim solitaire est mouillé

Et c'est son brame, n'est-ce pas, que j'entends.

46. IN, 217.

47. La section «poèmes shintô", jingika, est apparue pour la première fois dans le Goshûi waka shâ, la quatrième anthologie impériale, commandée en 1075 par l'empereur Shirakawa et achevée en 1086.

48. Sur le rapport entre poésie japonaise et shintô, voir Robert N. Huey, op. cit, p. 301-304.

49. MGK, Genkyû 2 (1205), III, 26.

50. Précisons toutefois que sur les treize poèmes composés par des dieux, seuls les trois premiers contiennent des mots de saison.

51. Il existe une traduction française de ce texte due à G. Renondeau, Contes d'Ise, Gallimard, 1969.

52. Il s'agit d'un poème qui apparaît dans l'Ise monogatari / Contes d'Ise (anecdote $\mathrm{n}^{\circ} 87$ ), le fameux texte de l'époque de Heian, où nous pouvons lire:

«[...] De bonne heure le matin les servantes de la maison sortirent pour ramasser le goémon rejeté par le flot et le rapportèrent à la maison. Elles l'entassèrent sur des plateaux montés sur des piquets et le couvrirent de feuilles de chêne. Sur les feuilles, [l'une d'elles] écrivit:

Watatsumi no Les algues

kazashi ni sasu to

Que le dieu des mers pour orner sa chevelure

iwahu momo Garde précieusement

kimi ga tame ni wa N'ont pas craint de venir ici woshimazarikeri

Parce que c'était pour vous.»

Nous reprenons ici la traduction de G. Renondeau, Contes d'Ise, Gallimard, Paris 1969, p. 138.

53. Il s'agit du poème anonyme, $\mathrm{n}^{\circ} 139$ dans la première anthologie impériale, le Kokin waka shû :

Satsuki matsu

Quand au cinquième mois

hanatachibana no

L'oranger sauvage

ka wo kageba

Exhale son parfum

muskashi no hito no

sode no ka zo suru

Il a la même fragrance

Que la manche de mon ancien amant!

54. Il s'agit d'un poème figurant dans les Contes d'Ise (trad. cit. p. 20) où nous pouvons lire:

«Il était jadis un homme. Il annonça à la dame de ses pensées un envoi d'algues, ajoutant: 


$\begin{array}{ll}\text { Omohi araba } & \text { Si vous m'aimiez } \\ \text { mugura no yado ni } & \text { Dans un pavillon délabré } \\ \text { ne mo shinan } & \text { Vous dormiriez [avec moi] } \\ \text { hijiki mono ni wa } & \text { Même si pour tapis } \\ \text { sode wo shitsutsu mo } & \text { Nous n'avions que nos manches. » }\end{array}$

55. Allusion, là encore, à un poèmes des Contes d'Ise (anecdote $n^{\circ} 13$, trad, cit. p. 34). Notons que l'auteur introduit de multiples jeux de mots qui obligent à juxtaposer deux traductions des trois premiers vers:
Musashi abumi
Comme aux étriers de Musashi
sasuga ni kakete
Fixés à leurs boucles
tanomu ni wa
On se fie
towanu mo tsurashi
Aux étriers de Musashi
tohu mo urusushi
Je me suspens malgré tout, éprise,
Et j'ai confiance en vous.
Si vous ne vous inquiétez de moi, j'en souffre
Si vous vous en inquiétez, c'est détestable.

56. Notons que le 28 du deuxième mois de cette même année Genkyû 2 (1205), est mort Fujiwara no Takanobu, le demi-frère de Teika, qui faisait également partie du Bureau de la poésie. On dit à Teika ( $M G K$, Genkyû 2, III, 28) «qu'à sa dernière heure son attitude fut d'une dignité remarquable; qu'il mourut assis en invoquant à haute voix le Bouddha, vêtu de blanc et tenant à la main le fil de cinq couleurs.»

57. Robert N. Huey, op. cit., p. 312.

58. Les poèmes composés à cette occasion sont appelés Nihongi kyôen waka ou «Poèmes japonais composés lors d'un banquet [suivant des leçons sur les] Chroniques du Japon »; il existe un recueil de ces poèmes.

59. MGK, Genkyû 2 (1205), III, 22.

60. $M G K$, Genkyû 2, III, 22.

61. $M G K$, Genkyû 2, II, 21.

62. IN, p. 219-220.

63. Alias princesse Ryôshi, fille de l'empereur Goshirakawa.

64. Il s'agit du prince Morinari, fils de Gotoba-in et futur empereur Juntoku.

65. Il s'agit sans doute des poèmes $n^{\circ} 709$ et 728 de notre anthologic. Le premier jour du rat de l'année, les courtisans allaient cueillir dans la nature de jeunes pousses de pin; il s'agissait là d'une célébration censée favoriser la longévité des personnes.

66. $M G K$, Genkyû 2 (1205), III, 29. Teika réitérera son admiration pour cette préface en Jôgen I (1207), III, 19, disant «qu'il ne faut pas en changer la moindre lettre ».

67. Il s'agit du Genkŷû shiika-awase qui eut lieu en Genkyû 2 (1205), le 15 du sixième mois. Huit poèmes de ce concours ont été inclus dans l'anthologie.

68. Nous suivons pour ce passage, corrompu dans l'original, l'interprétation proposée dans lenaga nikki, kôhon, kenkyû, sôsakuin, p. 277.

69. IN p. 223.

70. Ienaga décrit dans ses mémoires (IN, p. 265-266) la mort de Yoshitsune et l'émotion qu'elle suscita dans le milieu de la Cour. 
71. Nous pouvons lire dans le $M G K$, en date de Jôgen 1 (1207), VI, 8: «[...] Le regretté Yoshitsune me manque, je n'ai [plus] personne à qui demander conseil.»

72. Le Shinkokin waka shû est, à notre connaissance, la seule anthologie compilée collectivement pour laquelle nous sachions quel compilateur choisit chacun des poèmes séléctionnés. On trouvera ces indications en annexe de notre édition de référence de l'anthologie, p. 332-370.

73. Il s'agit de l'épouse du ministre des affaires suprêmes, morte avant son entrée en religion.

74. Il s'agit d'œuvres commandées afin d'être calligraphiées - seules certaines pièces le furent effectivement - sur les parois intérieures de la Chapelle des Quatre Dieux Rois, Saishô shi tennô-in, que Gotoba fit construire en 1207. Au sujet de cette manifestation, voir, par exemple, Huey (op. cit, p. 369-380).

75. Notre édition de référence, établie par Kubota Jun, contient précisément 1979 waka. On relève toutefois de légères différences entre les éditions.

76. Emmanuel Fraisse, Les Anthologies en France, PUF, « Écriture», Paris, 1997, p. 11.

\section{Bibliographie}

Éditions des sources:

lenaga nikki, kôhon, kenkyû, sôsakuin (ouvrage collectif publié par le groupe de recherches IENAGA NIKKI KENKYUKAI), Kazama shobô, 1985.

Ishida Yoshisada, SaTsuKawa Shûji (notes et commentaires), Ienaga nikki zenchûkai, Yûseidô, 1968.

ImAGAWA Fumio (notes et transcription en japonais classique), Meigetsuki-shô, Kawade shobô shinsha, 1986.

KuBota Jun (notes et commentaires), Shinkokin-wakashû, coll. Shinchô Nihon koten bungaku shûsei, Shinchôsha, 1979, 2 vol., réed. 1991 (vol. 1), 1992 (vol. 2).

Meigetsuki, Kokusho kankôkai, 1969, 3 vol.

\section{Etudes et traductions :}

ARIYOSHI Tamotsu, Shinkokin-wakashû no kenkyû, kiban to kôsei, Sanseidô, 1968.

FraISSE Emmanuel, Les Anthologies en France, Presses Universitaires de France, Paris, 1997.

Frank Bernard, Annuaire de l'École Pratique des Hautes Études, IVe section, Sciences historiques et philologiques 1976-1977.

Gotô Shigeo, Shinkokin-wakashû no kisoteki kenkyû, Hanawa shobô, 1968.

HERAIL Francine, Notes journalières de Fujiwara no Michinaga, ministre à la cour de Hei.an (995-1018), vol.1, Librairie Droz, 1987.

HuEy Robert N., The Making of Shinkokinshû, Harvard East Asian Monographs 208, Harvard University Press, 2002.

KONISHI Jin'ichi, Brower Robert H., MINER Earl, «Association and progression: Principles of Integration in Anthologies and Sequences in Japanese Court Poetry, A.D. 900-1350», Harvard Journal of Asiatic Studies, vol. 21 (déc., 1958), p. 67127. 
Michel Vieillard-Baron

RenOndeAu Georges (trad.), Contes d'Ise [Ise monogatari], Gallimard, coll. «Connaissance de l'Orient », Paris, 1969.

VIEILLARD-BARON Michel, Fujiwara no Teika (1162-1241) et la notion d'excellence en poésie, Théorie et pratique de la composition dans le Japon classique, Collège de France/Institut des Hautes Études Japonaises, Paris, 2001. 